\title{
Clinical Efficacy of Hybrid Surgery for Stanford Type A Aortic Dissection
}

\section{Jianjun $\mathrm{Gu}$ \\ Ziying Chen}

Department of Cardiac Surgery, Second Hospital of Hebei Medical University, Shijiazhuang, 050000, People's Republic of China
Correspondence: Ziying Chen

Department of Cardiac Surgery, Second Hospital of Hebei Medical University, 215

Heping West Road, Shijiazhuang, 050000,

Hebei Province, People's Republic of

China

Tel +86 3II 66002994

Email ziyingcv@163.com
Introduction: To evaluate the clinical efficacy of hybrid surgery for Stanford type A aortic dissection.

Methods: Twenty-two patients with Stanford type A aortic dissection were selected. All patients had completed or undergone hybrid surgery, including extracorporeal circulation, treatment of proximal anastomosis of ascending aorta and the distal anastomosis of the ascending aorta, management of the branch vessels on the arch, aortic endovascular repair. This study analyzed the time of surgery and awake, blood transfusion during surgery, patient's drainage, complications and CTA of aorta was re-examined about one month after operation during patients follow-up.

Results: All patients underwent the operation successfully. One patient died of renal failure after the operation. Two patients experienced postoperative neurological complications (anxiety and delirium). Renal function was abnormal in two patients, and one patient needed bedside blood filtration. The serum creatinine levels temporarily increased in seven patients. No stent migration was found during patient follow-up. There was no shift in the stents. The near end of the interlayer was well sealed, without leakage of contrast agent, and the false lumen near the stent was completely thrombosed. Compared with the pre-operative CTA, the true lumen was enlarged and the false lumen was reduced, and the false lumen was completely thrombosed in the proximal end and near the stent. Contrast media was seen in the false lumen.

Conclusion: One-stage hybrid surgery for Stanford type A aortic dissection can avoid deep hypothermic circulatory arrest, shorten operation time, reduce operation trauma, and reduce the incidence of postoperative complications. This treatment has a effective treatment effect in the short term. However, the limitations imposed by covered stent materials mean that the treatment's long-term effect is not yet clear, and further research is needed.

Keywords: aortic dissection, Stanford type A, one-stage hybrid surgery, postoperative complications, ascending aorta

\section{Introduction}

Aortic dissection occurs when an injury to the intima of the aorta allows blood to flow between the layers of the aortic wall, forcing the layers apart. Aortic dissection can quickly lead to death from insufficient blood flow to the heart or rupture of the aorta. ${ }^{1-3}$ Aortic dissection can be classified as either Stanford type A or Stanford type $\mathrm{B}$ according to the origin of the interlayer. Type A dissections originate from the ascending aorta, whereas Stanford type B dissections originate from the descending aorta and do not involve the ascending aorta. Stanford type A aortic dissection is a catastrophic disease that seriously endangers patients' physical and mental health. If 
not treated in time, $33 \%$ of patients die within 24 hours, and $36 \%-72 \%$ of patients die within 48 hours. $^{3}$

Acute Stanford type A (type I) aortic dissection should be treated by emergency operation following diagnosis. Surgery is the primary treatment for this disease, but the major surgical methods that are used can cause a wide range of injuries, and the operation requires complicated surgical procedures and deep hypothermic circulatory arrest. ${ }^{4-6}$ The traditional surgical method requires thoracotomy, replacement of the aortic arch or descending aorta under deep hypothermic circulatory arrest, and, potentially, stent elephant trunk implantation in the descending aorta. Because the operation is traumatic, there are many potential postoperative complications, and the mortality rate of the operation is very high. ${ }^{6}$ Hybrid technology and methods, which combine a multi-disciplinary procedure with minimally invasive techniques, provide a simpler and safer alternative to the complex and high-risk surgical methods used previously; this development is particularly advantageous for elderly patients and other patients that cannot tolerate deep hypothermic circulatory arrest. ${ }^{7-9}$ A hybrid cardiac surgical procedure is narrowly defined as a procedure that combines conventional surgery (including a skin incision) with intervention, using a catheter-based procedure guided by fluoroscopy, computerized tomography (CT) scan, or magnetic resonance imaging $(\mathrm{MRI})$ in a hybrid operating room without interruption. A wider definition of hybrid cardiac surgical procedures includes a clinically connected catheter intervention followed by a surgical procedure, with a time gap between the intervention and the surgery. ${ }^{10,11}$

This study was conducted to evaluate the clinical efficacy of hybrid surgery for Stanford type A aortic dissection.

\section{Materials and Methods}

\section{Subjects}

22 patients with Stanford type A aortic dissection were selected. The patients with aortic dissection had completed or undergone hybrid surgery. This study was conducted in accordance with the Declaration of Helsinki and approved by the ethics committee of the hospital (No.2016269). Written informed consent was obtained from all participants.

Inclusion criteria: (1) Patients diagnosed with Stanford type A aortic dissection, (2) patients older than 18 years of age, and (3) patients gave written informed consent. Exclusion criteria: (1) Patients had severe infection, (2) patients had severe heart, liver, or kidney dysfunction, (3) patients had severe coagulation dysfunction, and (4) patients had incomplete data.

\section{Preoperative Assessment}

The preoperative assessment of the patients was done using echocardiography, chest x-ray, electrocardiogram, international normalized ratio, arterial blood gas analysis, hemoglobin testing, full blood count, white blood cell count, fasting blood sugar, blood pressure measurement, CT scan, MRI, aortography, and D-dimer testing.

Once Stanford type A AD is diagnosed, in principle, active surgical treatment should be performed. Age is not contraindicated in acute Stanford Type A AD surgery. The results of IRAD study showed that the age of $>70$ years old was an independent risk factor for postoperative death of patients, but the case fatality rate was significantly lower than that of drug conservative treatment. Surgical treatment should be considered for acute Stanford type A AD patients of any age. According to the results of the national survey of cardiac surgeons in Canada, $97 \%$ of physicians believe that $>$ age 75 is not A contraindication for surgery at Stanford type A AD. However, for elderly patients, the treatment strategy should fully assess the condition of other organs in the body.

Acute Stanford type A AD combined with organ dysperfusion syndrome is the main risk factor affecting the treatment strategy and prognosis. At present, the consensus is that most of the patients with poor organ perfusion after aortic repair can be corrected. However, it is still controversial whether the patients with severe organ perfusion should undergo surgical operation. According to the 2014 ESC guidelines, preoperative coma, shock, stroke, and poor perfusion of coronary arteries and peripheral organs are risk factors affecting the prognosis of patients with Type A AD of Stanford, but should not be considered as contraindications for surgical operation. Previously, coma was considered to be an absolute contrainjection for acute Stanford Type A AD, but recently, El scholars believed that if the time from coma to operation was less than $5 \mathrm{~h}$, surgical operation could also be considered under good brain protection measures. At present, there are no reports of surgical outcomes in high-risk patients in China. Considering that China's transport system is not perfect and the diagnosis and treatment level varies from place to place, the expert committee believes that the Stanford type A $\mathrm{AD}$ patient who continues to be in coma is not suitable for surgical operation. Transient ischemic attack, transient body or language impairment caused by vascular involvement of the arm are not contraindicated in surgery. In 
addition, for patients with acute Stanford type A AD combined with intestinal ischemia, thoracic aortic surgery alone is often unable to effectively improve ischemia, and the postoperative mortality rate of patients is as high as $63 \%$. It has been reported abroad that abdominal aortic interventional endarterectomy combined with bronchial stent implantation can improve intestinal ischemia, but the overall rate of death and reintervention is still high. Combined with China's national conditions, the expert committee believes that severe intestinal ischemia is not suitable for surgical operation.

Indications of Hybrid Procedure: 1. > age: 60 years old or unable to tolerate long-term extracorporeal circulation surgery. 2. Chronic obstructive pulmonary disease. 3. Acute and chronic renal insufficiency. 4. Abnormal coagulation function.

Stent placement area: 1 . Stanford A surgically rebuilds the anchorage area to provide safe and adequate anchorage. Change to Stanford B sandwich. 2. Branch blood vessel reconstruction to ensure cerebral perfusion. 3. Interventional techniques for descending aorta reconstruction.

\section{Details of the Hybrid Procedure Extracorporeal Circulation}

All patients underwent aortic endovascular repair via the femoral and axillary arteries, combined with ascending aorta replacement under cardiopulmonary bypass. Each patient's body temperature was decreased to $32^{\circ} \mathrm{C}$, and the ascending aorta was blocked above the innominate artery. The ascending aorta was incised to the left and right coronary arteries to perfuse the cardioplegic solution. Cardiopulmonary bypass continued to reduce the body temperature to approximately $28^{\circ} \mathrm{C}$.

\section{Treatment of Proximal Anastomosis of Ascending Aorta}

Of the 22 patients, 16 had simple involvement of the aortic sinus without involvement of the coronary sinus, 5 had absence of the sinuses of valsalva and partial involvement of the right coronary sinus, and 1 had involvement of three coronary sinuses. In 16 of the patients, the ascending aorta was excised at $1 \mathrm{~cm}$ from the coronary artery opening, and the proximal stump of the aorta was made using two pieces of Bai Ren $\mathrm{Si}$ bovine pericardium, which was approximately $1 \mathrm{~cm}$ wide. The inner and outer linings were fixed with 4-0 slide suture in one circle.

Two patients underwent the David procedure and four patients underwent the Bentall procedure. After the aortic root was treated, four forked artificial blood vessels of 26/
28 were pruned, the proximal end was cut at $1.5 \mathrm{~cm}$ from the branch vessel, and the proximal end of each artificial blood vessel was anastomosed to the ascending aorta with continuous suture.

\section{Treatment of the Distal Anastomosis of the Ascending Aorta}

In all 22 patients, the ascending aorta was anastomosed to the artificial blood vessel at the proximal end of the innominate artery, and the aorta was clamped between the innominate artery and the left common carotid artery. At the same time, the innominate artery was blocked, the artificial blood vessel was closed to the innominate artery, and the aorta was anastomosed (hemiarch replacement). In 18 patients, bovine pericardium was used for the inner lining of the aortic root stump; in 4 patients, were made from the inner lining of the aortic stump. The artificial blood vessel and the distal aorta were closed using continuous suture (Table 1).

\section{Management of the Branch Vessels on the Arch}

The bypass of the branch vessels in the aortic arch was performed after the open heart beat. The distal end of the branch was blocked with blocking forceps, and the proximal end was clipped. The left subclavian artery, the left common carotid artery, and the innominate artery were blocked and anastomosed in order to reduce the time of cerebral ischemia. The $8 \mathrm{~mm}$ branch vessels at the farthest end of the four forked vessels were severed, and the two $10 \mathrm{~mm}$ branch vessels were anastomosed to the innominate artery and the left common carotid artery, respectively. An additional $8 \mathrm{~mm}$ artificial vessel was connected to the left subclavian artery.

\section{Aortic Endovascular Repair}

Once the operation of the aorta was complete, the cardiopulmonary bypass machine was stopped, and the extracorporeal circulation cannula was withdrawn. After the injected

Table I Preparation Method

\begin{tabular}{|l|l|l|}
\hline Age Group (Years) & Males & Females \\
\hline $65-66$ & 2 & 1 \\
$67-68$ & 12 & 3 \\
$69-70$ & 2 & 2 \\
Sub Total & 16 & 6 \\
\hline Total & $16+6=22$ \\
\hline Mean Age & $(65+70) / 2=67.5$ Years \\
\hline
\end{tabular}


protamine was neutralized, hemostasis was stopped, and the chest temporarily closed. The wound was then covered with a sterile pad, and an intravascular stent was prepared using digital subtraction angiography (DSA). The right femoral artery was cut or incised, and a catheter was inserted. A super-hard guide wire was inserted into the ascending aorta and a $30 \mathrm{~mm} \times 200 \mathrm{~mm}$ thoracic stent was implanted through the guide wire. The stent was covered at its proximal end, and it partially crossed $2 \mathrm{~cm}$ of the distal anastomosis of the artificial blood vessel. The contrast examination with DSA showed that the proximal break of the dissection was closed and that no contrast medium remained in the false lumen. The guide wire was removed and the femoral artery was repaired. After the chest was closed in a routine fashion, the patient was sent to the intensive care unit with tracheal intubation.

\section{Results}

\section{The General Characteristics of the}

\section{Patients}

22 patients with Stanford type A aortic dissection were selected, and all patients had undergone hybrid surgery. As shown in Tables 2 and 3, the patient group comprised 16 males and 6 females 65-70 years of age (mean age, $67 \pm$ 2.4 years) with a body mass of 57.0-93.0 kg (mean body mass, $76.9 \pm 12.9 \mathrm{~kg}$ ). Of the patient group, $18 \mathrm{had}$ a history of sudden chest pain, and severe tearing back pain, and 4 patients were admitted due to abdominal discomfort. All 22 patients had a history of hypertension with a duration of 1-12 years (mean duration, $6.5 \pm 4.6$ years). Echocardiography revealed floating intimal slices in the distal part of the ascending aorta in 21 patients and in the aortic arch in 1 patient. CT scans revealed 22 cases of Stanford type A aortic dissection with the ascending aorta and aortic arch involved, as well as 3 cases of false endovascular thrombosis of the ascending aorta. The onset of the disease was acute in all patients, with a duration of 6-67 hours (mean duration, $48.6 \pm 18.3$ hours). All patients underwent electrocardiographic examination to exclude acute coronary syndrome. The details of the preoperative photographs are given in Figure 1.

\section{Death After Surgery}

All patients underwent the operation successfully, One patient died of renal failure after the operation.
Table 2 Baseline Characteristics of Patients

\begin{tabular}{|l|c|}
\hline Age & $\mathbf{6 7 \pm 2 . 4}$ \\
\hline Male & 16 \\
Female & 6 \\
Body weight (Kg) & $76.9 \pm 12.9$ \\
Medical history (d) & $6.5 \pm 4.6$ \\
CTA & Stanford A Type I \\
Cardiopulmonary bypass time (min) & $180.0 \pm 25.0$ \\
Aortic block time (min) & $120.0 \pm 11.0$ \\
Operation time (h) & $9.0 \pm 1.5$ \\
Awake after operation (h) & $15.0 \pm 9.0$ \\
24hDrainage (mL) & $812.0 \pm 85.0$ \\
Intensive care unit stay time (d) & $5.0 \pm 2.0$ \\
Blood transfusion (mL) & $1700.0 \pm 320.0$ \\
Postoperative hospital stay (d) & $18.0 \pm 3.0$ \\
Smoking history (years) & $19.0 \pm 2.3$ \\
Drinking history (years) & $20.0 \pm 4.6$ \\
Hypertension & 18 \\
Diabetes & 2 \\
Coronary Heart Disease & 1 \\
Stroke & 3 \\
Marfan & 1 \\
Pericardial effusion & 11 \\
Pleural effusion & 2 \\
Lung infection & 2 \\
Liver insufficiency & 2 \\
Renal insufficiency & 1 \\
\hline
\end{tabular}

Table 3 Intraoperative Details

\begin{tabular}{|l|c|}
\hline Name of Operation & $\begin{array}{c}\text { Number of } \\
\text { Cases }\end{array}$ \\
\hline Stanford A & 22 \\
\hline $\begin{array}{l}\text { Ascending aorta replacement + total arch } \\
\text { replacement + elephant trunk implantation }\end{array}$ & 10 \\
\hline $\begin{array}{l}\text { Bentall surgery + total arch replacement + } \\
\text { elephant trunk implantation }\end{array}$ & 4 \\
\hline $\begin{array}{l}\text { Modified David surgery + total arch replacement } \\
+ \text { elephant trunk implantation }\end{array}$ & 2 \\
\hline $\begin{array}{l}\text { Ascending Master Replacement + Total Arch } \\
\text { Replacement + Elephant Trunk Stent Implantation }\end{array}$ & 6 \\
\hline
\end{tabular}

\section{Details During Surgery}

The cardiopulmonary bypass time was 80-116 minutes, and the aortic clamping time was 28-67 minutes. Twenty patients were awake in the intensive care unit within 6 hours. The blood consumption during and after the operation was 4-8 units of red blood cells, 0-4 units of platelets, 


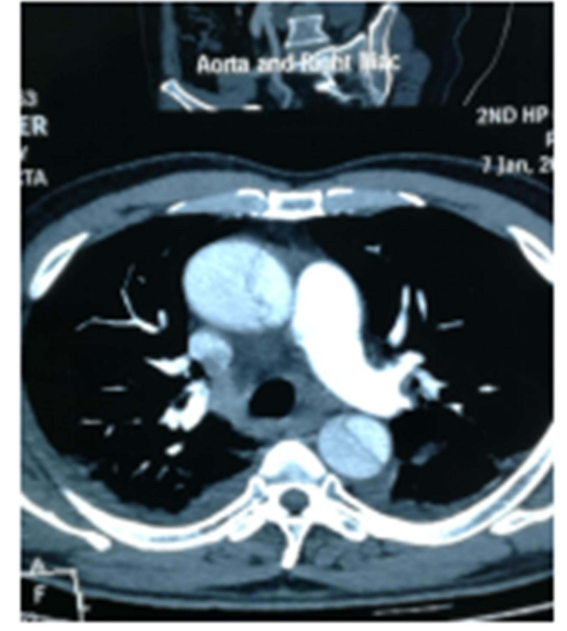

\section{Preoperative Photo CTA 1}

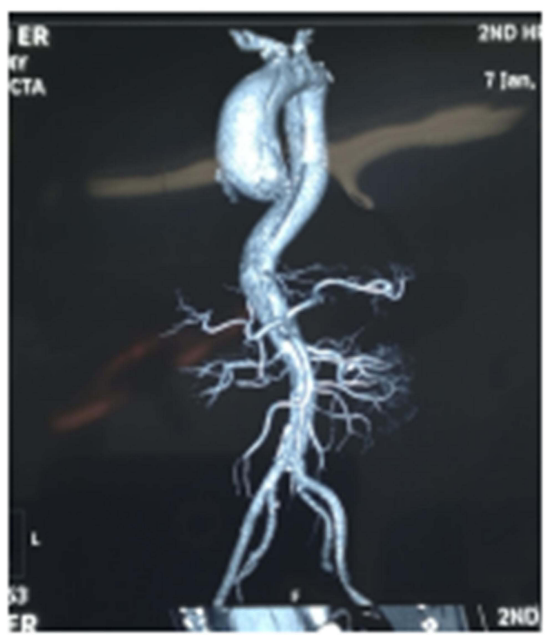

Preoperative PhotoCTA 3

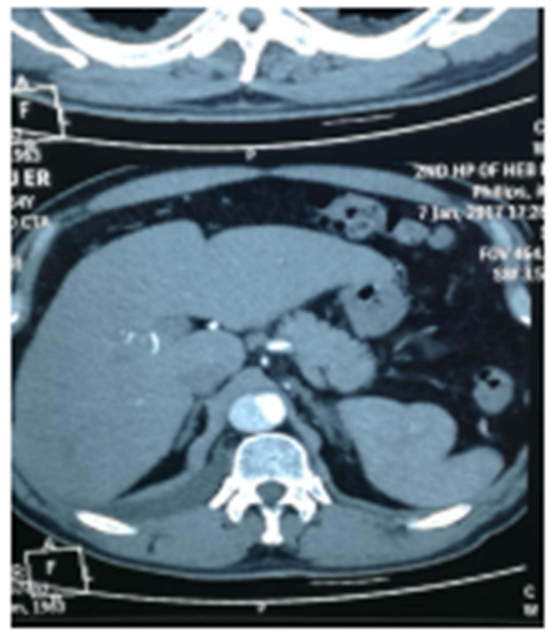

Preoperative Photo CTA 2

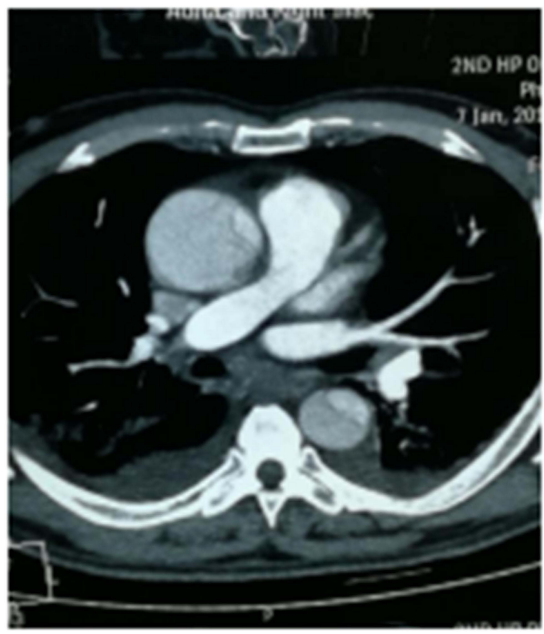

Preoperative Photo CTA 4

Figure I Preoperative photos and descriptions.

and 0-20 units of cryoprecipitate. The first 24-hour drainage was $325-575 \mathrm{~mL}$, with a total discharge of 650 $1350 \mathrm{~mL}$. The details of the operative photographs are listed in Figure 2.

\section{Complications After Surgery}

As shown in Tables 4, 18 patients were extubated within 48 hours after operation. Extubation was performed 7 days after operation in one patient and 10 days after operation in another patient. Postoperative neurological complications (anxiety and delirium) occurred in two patients. Renal function was abnormal in two patients, and one patient needed bedside blood filtration. The serum creatinine of seven patients increased temporarily. Postoperative pleural effusion occurred in two patients but improved after Thoracentesis.

\section{Clinical Follow-Up}

$\mathrm{CT}$ angiography scans of the aorta were re-examined during the patients' follow-up appointments, approximately one month after the operation. No stent migration was found. The near end of the interlayer was well sealed with no leakage of the contrast agent, and the false lumen near the stent was completely thrombosed. Compared with the results of the pre-operative CT angiography scans, the true lumen was enlarged, and 

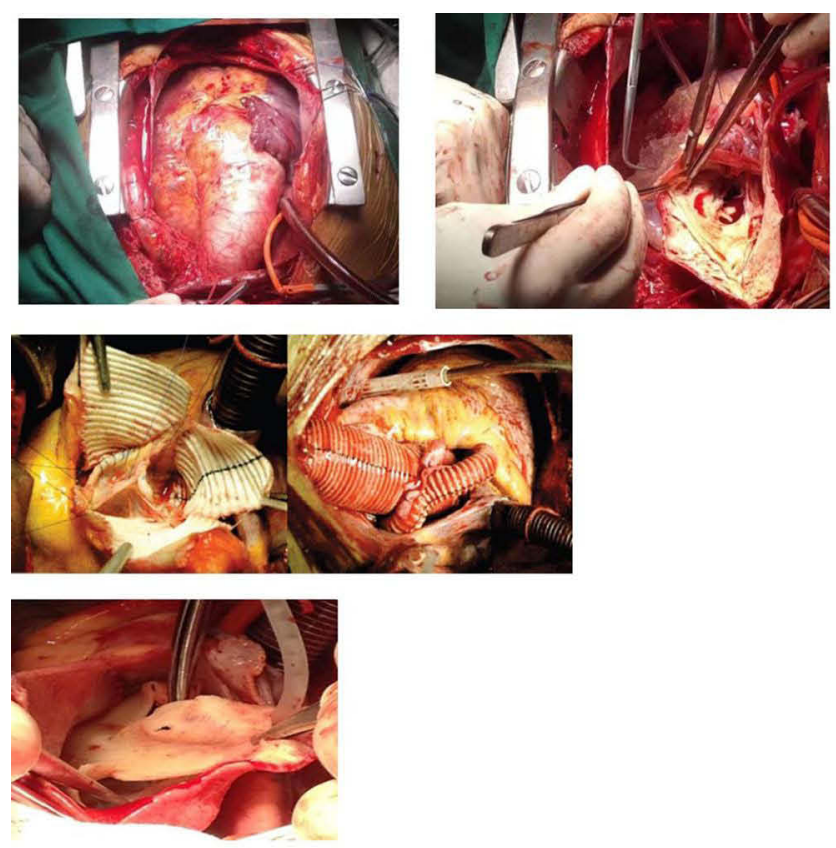
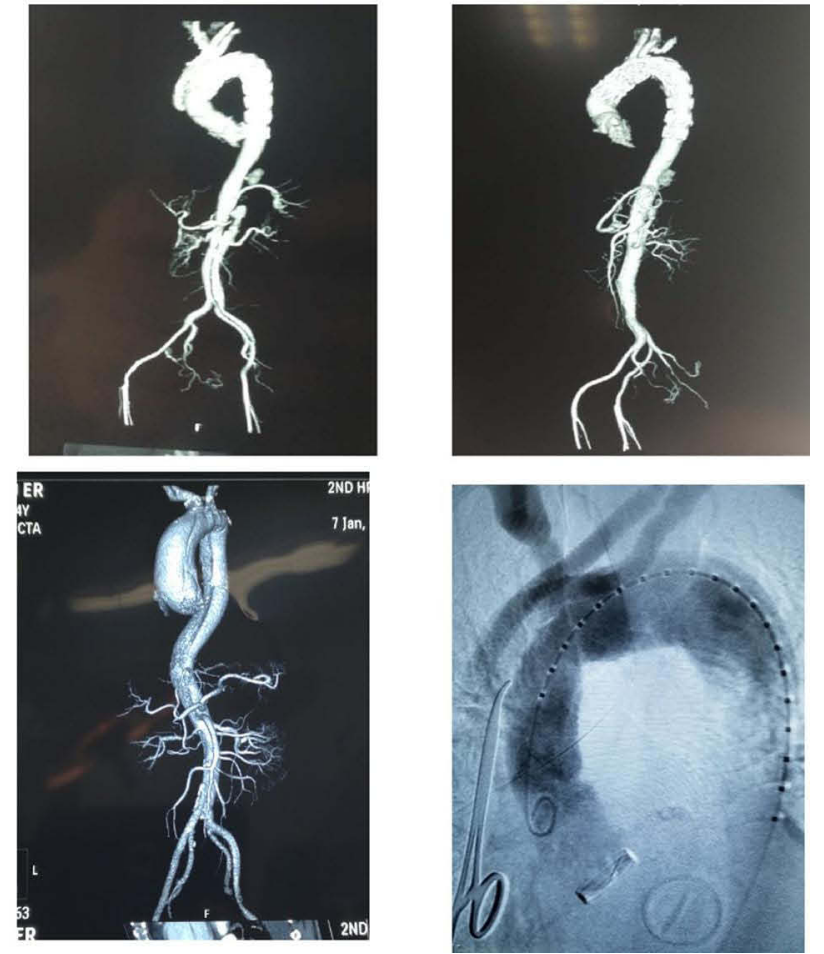

Figure 2 Operative photos.

the false lumen was reduced. In addition, the false lumen was completely thrombosed, both in its proximal end and close to the stent, and contrast media was seen in the false lumen. The details of the postoperative photographs are given in Figures 3-5

\section{Discussion}

The use of hybrid surgery to treat aortic disease has become increasingly widespread, making endovascular repair simpler in China. Hybrid surgery is primarily performed between the bypass flows of 3-branch vessels to treat aortic lesions involving the aortic arch. Hybrid surgery is used less frequently to treat lesions involving the

Table 4 Post-Surgery Complications

\begin{tabular}{|l|l|}
\hline Nervous System Complications & \\
Paraplegia & 2 \\
Acute cerebral infarction & 2 \\
Transient brain dysfunction & $\mathrm{I}$ \\
\hline Acute liver injury & $\mathrm{I}$ \\
Acute kidney injury & 2 \\
Hoarse voice & 4 \\
Lung infection & 2 \\
Acute pulmonary embolism & 0 \\
\hline
\end{tabular}

ascending aorta and the entire aorta: Only sporadic instances have been reported, with the highest number coming from Fuwai Hospital in Beijing. In comparison with the traditional methods of operation, such as contemporary series or use of FET technique, hybrid surgery involves a shorter operation time and can reduce operation trauma, shorten the length of the postoperative hospital stay, decrease the number of units of blood that must be transfused, and provide a more satisfactory early therapeutic effect. ${ }^{12,13}$

Several aspects of the specific surgical procedures that were used are of particular note. First, double cannulation of the right axillary femoral arteries was performed. As a result, even if the lesion was found to be complex and require special treatment, it can be changed to routine operation without affecting the perfusion of the head. ${ }^{14-}$ ${ }^{16}$ Second, when aortic root and proximal anastomosis were performed, it was necessary to shift the branch vessels as close to the proximal segment as possible to ensure that the distal artificial blood vessels had enough area for anchor and support. After being strengthened by the sandwich method, the distal aortic anastomosis was anastomosed with the artificial blood vessel. In the absence of an artificial vessel lining, it is very difficult to perform anastomosis on the walls of the diseased aorta, and the 


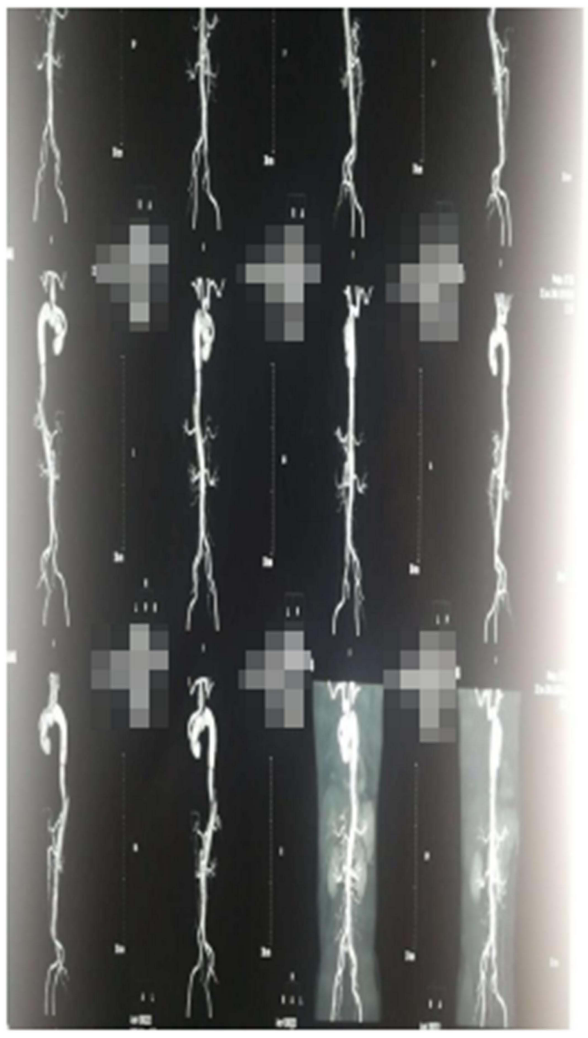

POSTOPERATIVE CTA 1

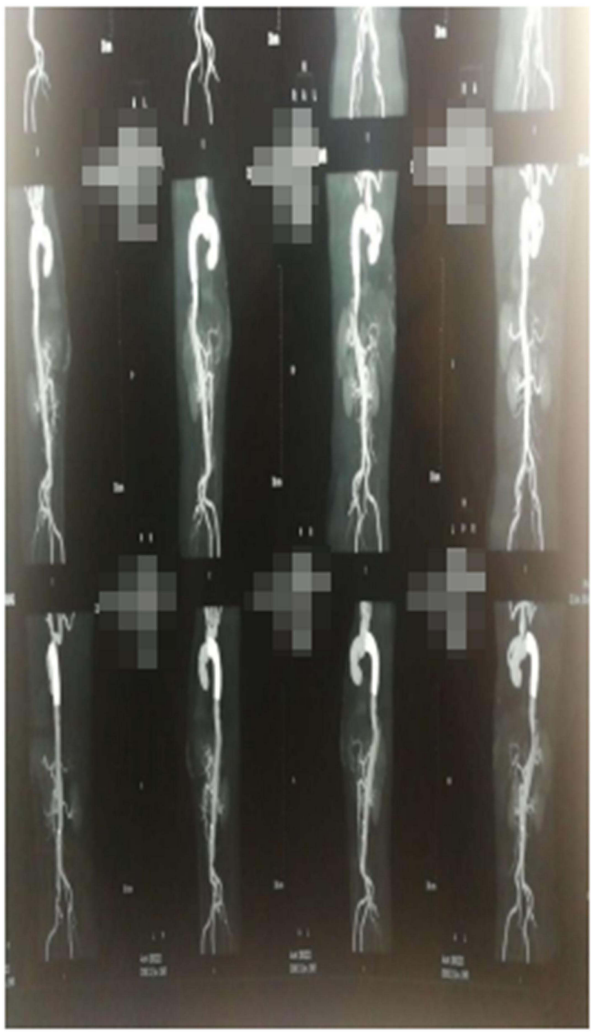

POSTOPERATIVE CTA 2

Figure 3 Postoperative photos I-2.

intima of the aorta can tear easily. Reinforcement of the anastomosis prevented otherwise inevitable bleeding. Distal anastomosis was primarily performed on the anterior $1 \mathrm{~cm}$ of the innominate artery; this seemed to be more appropriate for routine deep hypothermia surgery.

Third, although one-stage hybrid surgery avoids deep hypothermic circulatory arrest, reduces surgery time, and reduces surgical trauma, the procedure has several potential drawbacks, namely the covered stent vessels, the attachment of the stent to the aorta, and the long-term life of the stent. ${ }^{17,18}$ To address the first point, the first thoracic aortic stent system was adopted, since it adapts well to the shape of the arch and has good plasticity. Regarding the long-term life of the stent, although stents have been used for longer than 10 years in patients with Stanford type B aortic dissection, stents are primarily located at the distal end of the arch and descending aorta; by contrast, Stanford type A aortic dissection requires a stent that can cover the entire aortic arch. ${ }^{19,20}$ At present, there are no clear data concerning the time of stent placement. Given the current material limitations, the most cautious approach is to use this procedure primarily with older patients. Patients with severe aortic dissection and a high risk of bleeding can improve their chances of survival by undergoing this procedure.

For younger patients that do not have severe neurological complications or coagulation disorders, the use of traditional deep hypothermic circulatory arrest surgery should still be appropriate. However, when the branch of the arch is completed to branch off, there can be long-term complications in the form of stent vascular rupture, although endovascular repair is an alternative option in such cases. Another unavoidable issue is the much higher cost of treatment associated with one-stage hybrid surgery using stents. For high-risk patients, hybrid surgery can offer a clear improvement in the cost of treatment because it can shorten the length of hospitalization and reduce bleeding and other complications. However, hybrid surgery does not offer a comparable financial advantage to patients with a better preoperative condition.

The results of this study demonstrate that, for Stanford type A aortic dissection, one-stage hybrid surgery is superior to traditional surgical methods that involve replacement of the ascending aorta, total arch replacement, and 


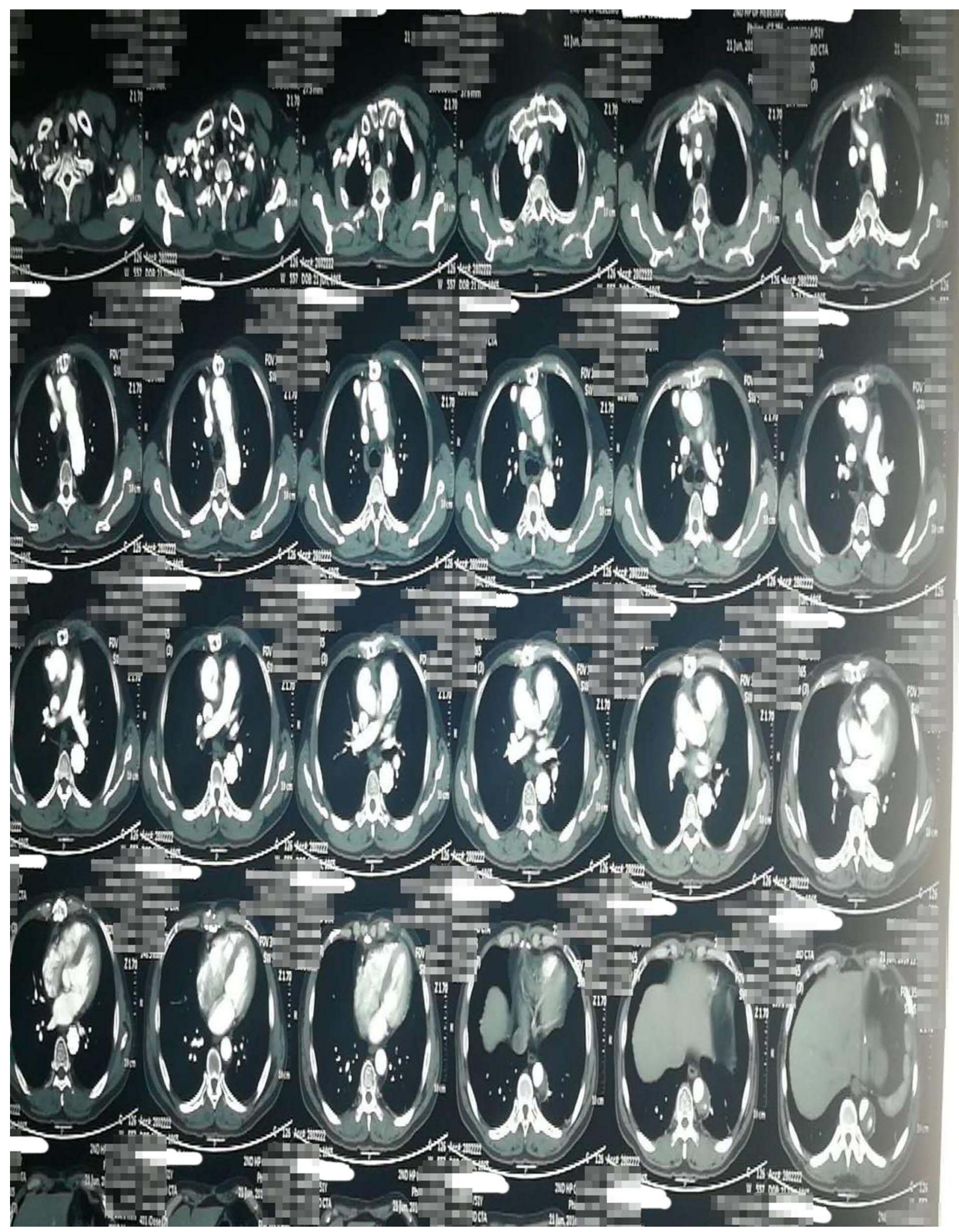

Figure 4 Postoperative photos 3. 


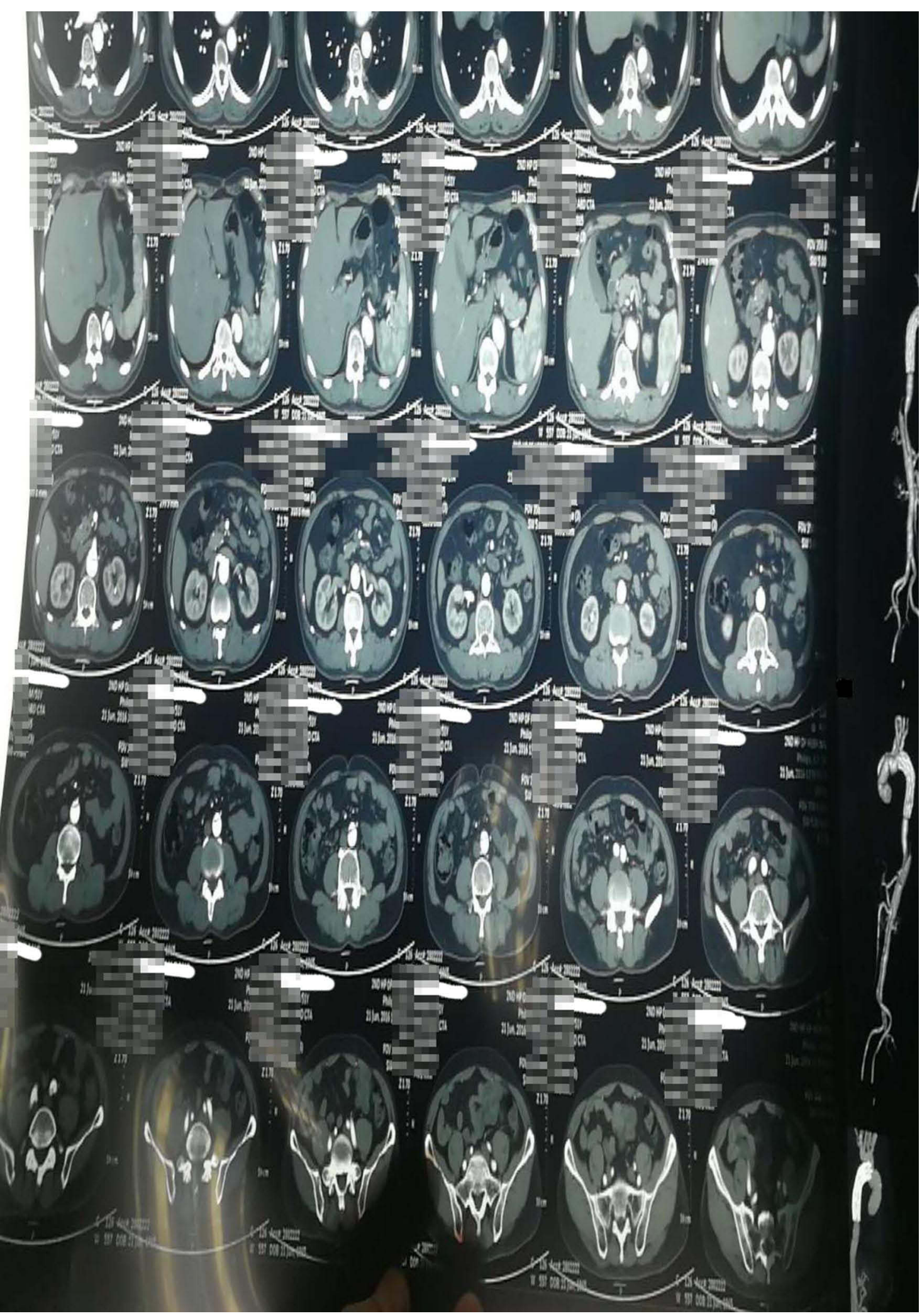

Figure 5 Postoperative photos 4. 
elephant trunk stenting. The use of hybrid surgery to treat aortic dissection is promising for elderly patients and other patients that cannot tolerate deep hypothermic circulatory arrest for longer periods. For other patients, however, the traditional surgical approach may still be more appropriate for the treatment of Stanford type A aortic dissection. The study found that hybrid surgery drastically reduced the mortality rate of patients with Stanford type A aortic dissection. Comparing the patient group in terms of sex, it appears that Stanford type A aortic dissection is more common in men than in women of the same age group. In addition to showing the effects of age and sex, the results of the study also show that untreated hypertension is the most common cause of Stanford type A aortic dissection.

This study had several limitations. First, it was not a randomized controlled trial. Second, it was only a singlecenter trial, and the sample size was limited. Third, the clinical follow-up was short, and longer-term follow-up will be necessary to determine the long-term clinical prognosis, including endoleaks, new distal dissections, stent migration.

\section{Conclusion}

One-stage hybrid surgery for Stanford type A aortic dissection can avoid deep hypothermic circulatory arrest, shorten operation time, reduce operation trauma, and reduce the incidence of postoperative complications. This treatment has a effective treatment effect in the short term. However, the limitations imposed by covered stent materials mean that the treatment's long-term effect is not yet clear, and further research is needed.

\section{Ethical Statement}

This study was conducted in accordance with the Declaration of Helsinki and approved by the ethics committee of Second Hospital of Hebei Medical University. All participants had signed the informed consent.

\section{Disclosure}

All of the authors had no any personal, financial, commercial, or academic conflicts of interest separately.

\section{References}

1. Vallejo N, Rodriguez-Lopez JA, Heidari P, et al. Hybrid repair of thoracic aortic lesions for zones and in high-risk patients. $J$ Vasc Surg. 2012;55(2):318-325. doi:10.1016/j.jvs.2011.08.042
2. Ucak A, Onan B, Inan BK, Temizkan V, Ugur M, Yilmaz AT. Hybrid repair of an acute type $\mathrm{B}$ dissection with subclavian to subclavian bypass and stent-grafting. JCard Surg. 2010;25(3):336-339.

3. Szeto WY, Bavaria JE. Hybrid repair of aortic arch aneurysms: combined open arch reconstruction and endovascular repair. Semin Thorac Cardiovasc Surg. 2009;21(4):347-354. doi:10.1053/j. semtcrs.2009.11.007

4. Jing H, Li DM, Hu XN. Hybrid surgery for the treatment of dissection of the aorta. Chin J Chest Cardiol. 2011;27(6):227-230.

5. Orihashi K. Malperfusion in acute type a aortic dissection: unsolved problem. Ann Thorac Surg. 2013;95(5):1570-1576. doi:10.1016/j. athoracsur.2013.02.025

6. Wu Haiwei, Jing Hua, Li Demin, et al. The use of a contralateral Atype aortic dissectionNeuroprotection of the nervous system during hybrid surgery.Chin J Cardiac Surgery. 2012,28(8):459-463.

7. Wei YZ, Chang Q, Yu CT, et al. Replacement of aortic arch and early follow-up results of one-stage hybrid surgery. Chin J Cardiac Surg. 2012;27(6):342-348.

8. Li G. Prof Shijiang Zhang: the development of hybrid surgery in China. J Thorac Dis. 2014;6(9):1368-1370.

9. Xydas S, Mihos CG, Williams RF, et al. Hybrid repair of aortic arch aneurysms: a comprehensive review. J Thorac Dis. 2017;9(Suppl 7): S629-S634. doi:10.21037/jtd.2017.06.47

10. Borst HG, Walterbusch G, Schaps D. Extensive aortic replacement using "elephant trunk" prosthesis. Thorac Cardiovasc Surg. 1983;31 (01):37-40. doi:10.1055/s-2007-1020290

11. Safi HJ, Miller CC 3rd, Estrera AL, et al. Staged repair of extensive aortic aneurysms: long-term experience with the elephant trunk technique. Ann Surg. 2004;240(4):677-684. doi:10.1097/01. sla.0000140756.30517.1b

12. Tian DH, Wan B, Di Eusanio M, Black D, Yan TD. A systematic review and meta-analysis on the safety and efficacy of the frozen elephant trunk technique in aortic arch surgery. Ann Cardiothorac Surg. 2013;2(5):581-591. doi:10.3978/j.issn.2225-319X.2013.09.07

13. Nollert G, Wich S, Figel A. The cardiovascular hybrid OR-clinical \& technical considerations. Cardiothorac Surg Netw. 2011.

14. Amr R, Ahmed A. Cannulation of innominate artery as an alternative to axillary artery in repair for type A aortic dissection. J Egypt Soc Cardio Thorac Surg. 2018;26(2):83-88. doi:10.1016/j. jescts.2018.05.004

15. Gegouskov V, Manchev G, Danov V, Stoitsev G, Iliev S. Direct cannulation of ascending aorta versus standard femoral artery cannulation in acute aortic dissection type A. Heart Surg Forum. 2018;21 (3):E139-E144. doi:10.1532/hsf.1956

16. Arpit T, Elvina W, Manu M. Experience with the axillary artery as an arterial cannulation site in patients with Type A aortic dissection. Heart Lung Circ. 2018;27:S536-S537. doi:10.1016/j.hlc.2018.04.098

17. Yip HC, Chan YC, Qing KX, Cheng SW. Retrograde type A dissection following hybrid supra-aortic endovascular surgery in high risk patients unfit for conventional open repair. $J$ Cardiovasc Surg. 2018;7(suppl):S34-S34.

18. Uehara K, Matsuda H, Matsuo J, et al. Aortic root surgery after acute type A aortic dissection repair. Heart Lung Circ. 2018;27:S538S539. doi:10.1016/j.hlc.2018.04.104

19. Hashiyama N, Goda M, Uchida K, et al. Stanford type B aortic dissection is more frequently associated with coronary artery atherosclerosis than type A. J Cardiothorac Surg. 2018;13(1):80. doi:10.1186/s13019-018-0765-y

20. Morrow KL, Johnson DJ, Shevitz AJ, Campos PR, Kashyap VS, Powell A. Clinical outcomes and false-lumen thrombosis following stanford Type-A and Type-B aortic dissection. Ann Vasc Surg. 2018;48:23-24. doi:10.1016/j.avsg.2018.01.041 


\section{Publish your work in this journal}

Risk Management and Healthcare Policy is an international, peerreviewed, open access journal focusing on all aspects of public health, policy, and preventative measures to promote good health and improve morbidity and mortality in the population. The journal welcomes submitted papers covering original research, basic science, clinical \& epidemiological studies, reviews and evaluations, guidelines, expert opinion and commentary, case reports and extended reports. The manuscript management system is completely online and includes a very quick and fair peer-review system, which is all easy to use. Visit http://www.dovepress.com/testimonials.php to read real quotes from published authors.

Submit your manuscript here: https://www.dovepress.com/risk-management-and-healthcare-policy-journal 